\title{
Operation and Control of Microgrids Using IoT (Internet of Things)
}

\author{
Dipta Voumick, Prince Deb, Mohammad Monirujjaman Khan \\ Department of Electrical and Computer Engineering, North South University, Dhaka, Bangladesh \\ Email:dipta.voumick@northsouth.edu,prince.deb@northsouth.edu,monirujjaman.khan@northsouth.edu
}

How to cite this paper: Voumick, D., Deb, P. and Khan, M.M. (2021) Operation and Control of Microgrids Using IoT (Internet of Things). Journal of Software Engineering and Applications, 14, 418-441.

https://doi.org/10.4236/jsea.2021.148025

Received: July 3, 2021

Accepted: August 23, 2021

Published: August 26, 2021

Copyright $\odot 2021$ by author(s) and Scientific Research Publishing Inc. This work is licensed under the Creative Commons Attribution International License (CC BY 4.0).

http://creativecommons.org/licenses/by/4.0/

\begin{abstract}
The current microgrid power management system is undergoing a significant and drastic overhaul. The integration of existing electrical infrastructure with an information and communication network is an inherent and significant need for microgrid classification and operation in this case. Microgrid technology's most important features: 1) Full duplex communication; 2) Advanced metering infrastructure; 3) Renewable and energy resource integration; 4) Distribution automation and complete monitoring, as well as overall power system control. A microgrid's communication infrastructure is made up of several hierarchical communication networks. Microgrid applications can frequently be found in numerous aspects of energy consumption. Because it provides a spontaneous communicational network, the Internet of Things plays a fundamental and crucial role in Microgrid infrastructure. This paper covers the deployment of a comprehensive energy management system for microgrid communication infrastructure based on the Internet of Things (IoT). This paper discusses microgrid operations and controls using the Internet of Things (IoT) architecture. Microgrids make use of IoT-enabled technologies, in conjunction with power grid equipment, which are enabling local networks to provide additional services on top of the essential supply of electricity to local networks that operate in parallel with or independently of the regional grid. Local balancing, internal blockage management, and request for support marketplace or grid operator activities are examples of auxiliary services provided by the microgrid that can add value to each end-user and other true stakeholders. Different technologies, architectures, and applications that use IoT as a key element with the main purpose of preserving and regulating innovative smart microgrids in accordance with modern optimization features and regulations are designed to update and improve efficiency, resiliency, and economics.
\end{abstract}

\section{Keywords}

IoT, Micro Grid, Operation, Control, Smart Meter 


\section{Introduction}

For the US Department of Energy, the Microgrid Exchange Collection, an ad hoc collection of research and deployment specialists, created the following widely recognized definition:

"A microgrid is a collection of interconnected loads and distributed energy resources that operate as a single controllable entity in relation to the grid and are contained within well-defined electrical boundaries. A microgrid may connect to the grid and disconnect from it, allowing it to function in grid-connected and island modes [1].”

Three prerequisites are included in this description: 1) The microgrid's neighborhood can be separated from the rest of the distribution system; 2) The resources linked to a microgrid are managed by one another rather than by remote resources; 3) The microgrid can operate whether or not it is connected to the larger grid. There is no mention of the scale of distributed energy resources or the technologies that will or should be implemented in the definition [2].

For balancing local loads and achieving economic advantages, microgrids have specific control needs and techniques. According to the agreement, microgrid controllers must have the following functional characteristics: Present the microgrid to the utility grid as a single self-contained entity capable of providing frequency control (similar to a synchronous generator system); avoiding power flow exceeding line ratings; regulating voltage and frequency within acceptable bounds during islanding; dispatching resources to ensure energy balance; islanding smoothly; safely reconnecting and resyncing [3]. Microgrids can be regulated in the same way as the main grid, that is, using a three-level hierarchical control system. Primary and secondary frequency and voltage regulation are typically performed by a Microgrid Central Controller (MGCC) that sends explicit instructions to distributed energy resources, or in a decentralized manner, such as CERTS, where each resource responds to local conditions [4].

Furthermore, microgrids typically include a tertiary control layer to enable economic and optimization operations for the microgrids, which is primarily focused on managing battery storage, distributed generation scheduling and dispatch, and managing electricity import and export between the microgrid and thus the utility grid. In two European microgrids, one on the Greek island of Kythnos and the other in the German "Am Steinweg" project, hierarchical control systems that regulate electricity inside a microgrid and mediate exchanges with the main grid are installed using a "multi-agent system" method. Increasingly, microgrid research and development is that concentrates on adding intelligence to optimize operational controls and market participation [5]. The ability of microgrids to improve the resiliency and reliability of "critical facilities" such as transportation, communications, beverage and waste treatment, health care, food, and emergency response infrastructure has been the main driver of microgrid development in the United States. The Northeastern United States is one of the main areas of activity, where aging infrastructure and regular extreme 
weather events have caused billions of dollars in damages in recent years. As a result, states are looking at the possibility of expanding microgrids beyond essential facilities to service entire towns, and they've started sponsoring demonstration projects [6]. The "New York Prize", a \$40 million competition to assist towns on the path from feasibility studies to implementation, is the most famous example of state backing for community microgrids. States in the United States are also considering microgrids as a method to swap out retiring generating capacity and ease congestion in the transmission and distribution system [7].

Figure 1 shows the general components for microgrid system. Several storage systems, Non-renewable power sources and renewable power sources have been used as energy sources.

More important drivers of microgrid activity have been climate change and the necessity to incorporate increasing volumes of renewable electric energy generated into the grid. Climate scientists have also concluded that, by 2050, human society must reduce the proportion of electricity produced by burning fossil fuels from $70 \%$ to under $20 \%$ to avoid a global average temperature rise exceeding $2^{\circ} \mathrm{C}$ above pre-industrial levels, which is currently accepted as the threshold between "safe" and "dangerous" climate change. Decentralized, intermittent, and non-dispatchable energy resources are scaling up to fill this gap, making them difficult to incorporate into a grid intended for a one-way flow of power from centralized produced plants to consumer demands. Patchy renewables combined with co-located as well as flexible loads and storage technologies in microgrids allows for local supply and demand balancing, making broad

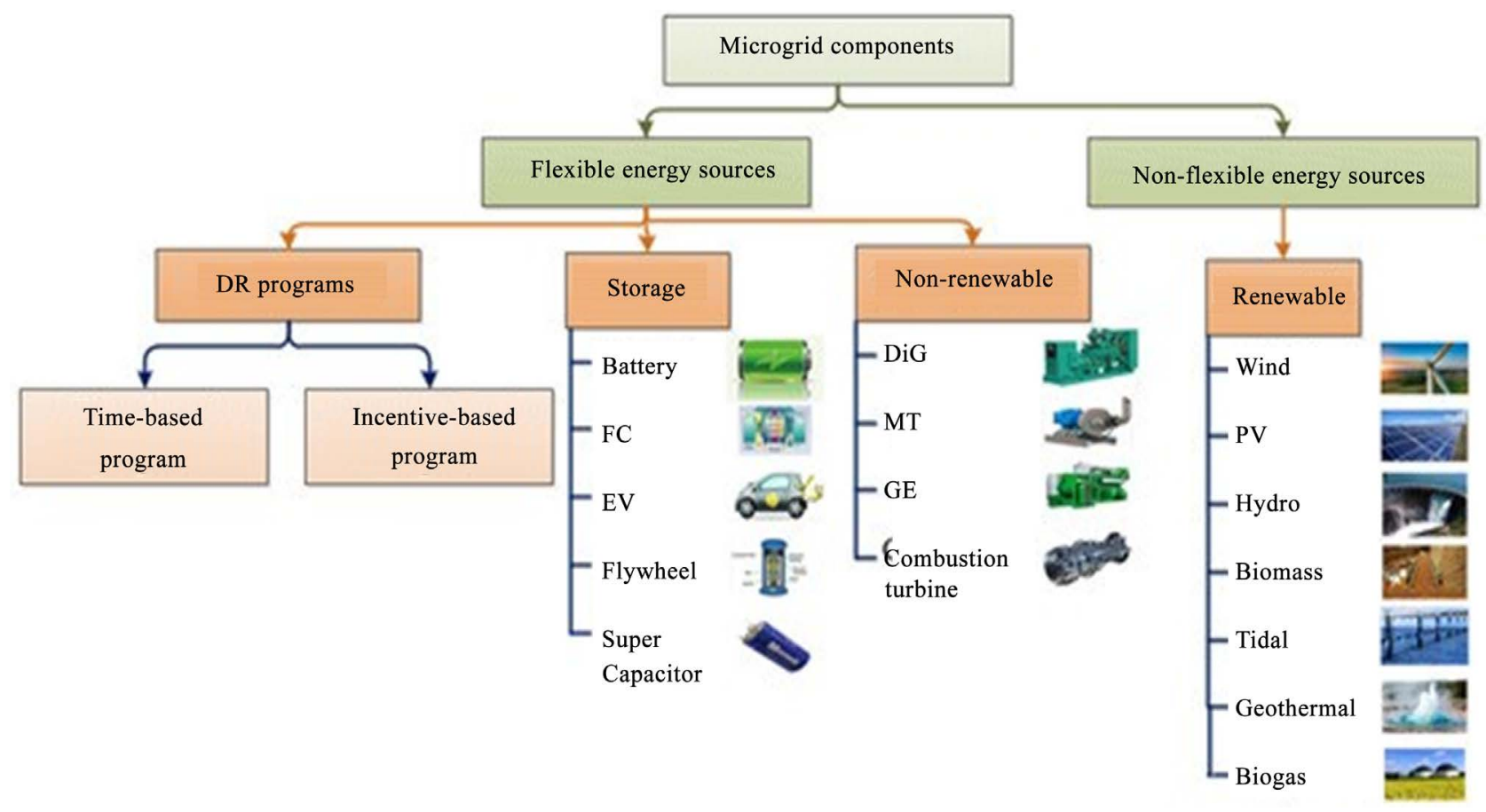

Figure 1. Components of Microgrid. 
renewable deployment more feasible. To the distribution utility, each microgrid appears as a small power producer or consumer, with the ability to change or vary the net load profile in ways that benefit the main grid the greatest [8]. Microgrid fuel savings and subsidiary grid services are key components of the business case in both locations, despite many disparities in the importance assigned to durability and exhalation. With the support of IoT, new research is being compiled to build microgrids using advanced analytical techniques in order to optimize these advantages over a broad marginal range of parameters, including land usage, water consumption, employment, $\mathrm{CO}$ emissions, investment expenses, and energy cost [9]. Microgrids are defined by the International Council on Large Electric Systems as an electricity distribution system that contains loads and distributed energy resources such as distributed generators, spontaneous storage devices, or controllable loads that will operate in a controlled and coordinated manner, either while connected to the main power network or while islanded in a telecommunications network. Following Figure 2, here the overall microgrid system is shown with a vivid diagram.

While much has been written about the idea and advantages of microgrids, real-world examples of microgrids in operation can also teach us something. A comprehensive list of existing, experimental, and simulated microgrid systems may be found here. According to Navigant Research, which has been tracking microgrid deployment since 2011, The United States has been one of the historical leaders in deployed capacity; however, at this time, The United States and Asia have roughly equal capacity in terms of operating, microgrids under development, and proposed microgrids, with each having 42 percent of the market. Europe has $11 \%$ of the capacity, Latin America has $4 \%$, while the Middle East and Africa have only $1 \%$ of the capacity. In 2015, total capacity was at $1.4 \mathrm{GW}$, and by 2024 , it is predicted to reach either $5.7 \mathrm{GW}$ or $8.7 \mathrm{GW}$, depending on the scenario. As of Q1 2016, Navigant divides the microgrid market into the following groups based on proportion of total installed power capacity: Remote (54\%),

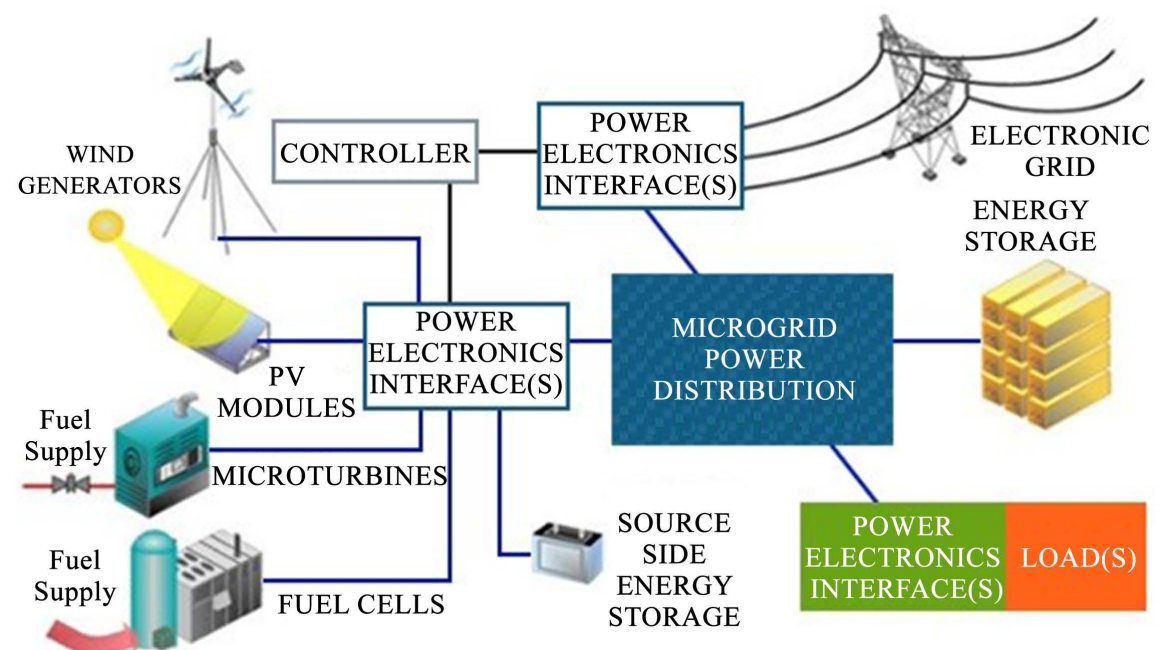

Figure 2. Overall microgrid system. 
Commercial/Industrial (5\%), Community (13\%), Utility Distribution (13\%), Institutional or Campus-based (9\%), and National guard (6\%). It should also be mentioned that Navigant Researches does not track pure diesel-generator-based remote microgrid systems, which must contain at least one renewable producing power source in order to be evaluated. Because it is not feasible to provide a complete list of diverse microgrid applications here, we will highlight a few [10].

\section{Operation of Microgrid}

The Internet of Things (IoT) is allowing businesses to construct smart grids, also known as microgrids, at a lower cost. Organizations can make better use of their power by leveraging this option to disconnect and function on the grid or in island mode. Due to the high integration technique of fashionable technology within a microgrid system, it's difficult to properly operate all the diversity of devices placed during a microgrid using a logically coordinated way. Possible ranked management of microgrids is mentioned. The operational characteristics and classifications of a microgrid area unit are completely different from rate with smaller generation inertia, total variable power generation thanks to RES integration, low fault currents once islanded, and so on. The first management in this framework is for dominant decigram units to have virtual inertias and regulate output electrical phenomena. Secondary management is in charge of correcting the first management loop's steady-state frequency and voltage magnitude mistakes [11]. Superior management is established for facility energy management based on a variety of factors. Such as microgrid stability, environmental issues and concerns, and so on. As a result, in order to achieve strong coordination of this ranking system, a centralized microgrid management method is being developed, in which three controllers are primarily used to control; Microgrid central controller, DER distributed controllers, and Distribution management system DCs work at the device level in the primary and secondary management area units, which are designed to ensure that each DER operates correctly. In microgrids, there are two levels of inertia, which means that each DC must be rapid and dependable in order to keep voltages, frequencies, and power flows within acceptable tolerances and to adjust in real-time to unknown and variable hundreds and network conditions [12]. The microgrid's facility management is decided at the MGCC level of tertiary management based on information from DERs active facility, load demand, and storage requirements. The inter-change of power and energy references or setpoints sent to the DERs and hundreds is enabled by a 2-method communication between the Microgrid management criteria and the DCs, while each individual DC ensures that the facility reference from the central management level is reached. Overall microgrid demands and stabilizing needs are appropriately met at the DMS level. As a result, the MGCC is primarily responsible for microgrid facility optimization, as well as the best management strategies to be conferred in the following section and area unit produced during this entity [13]. Figure 3 shows micrgrid operation. 


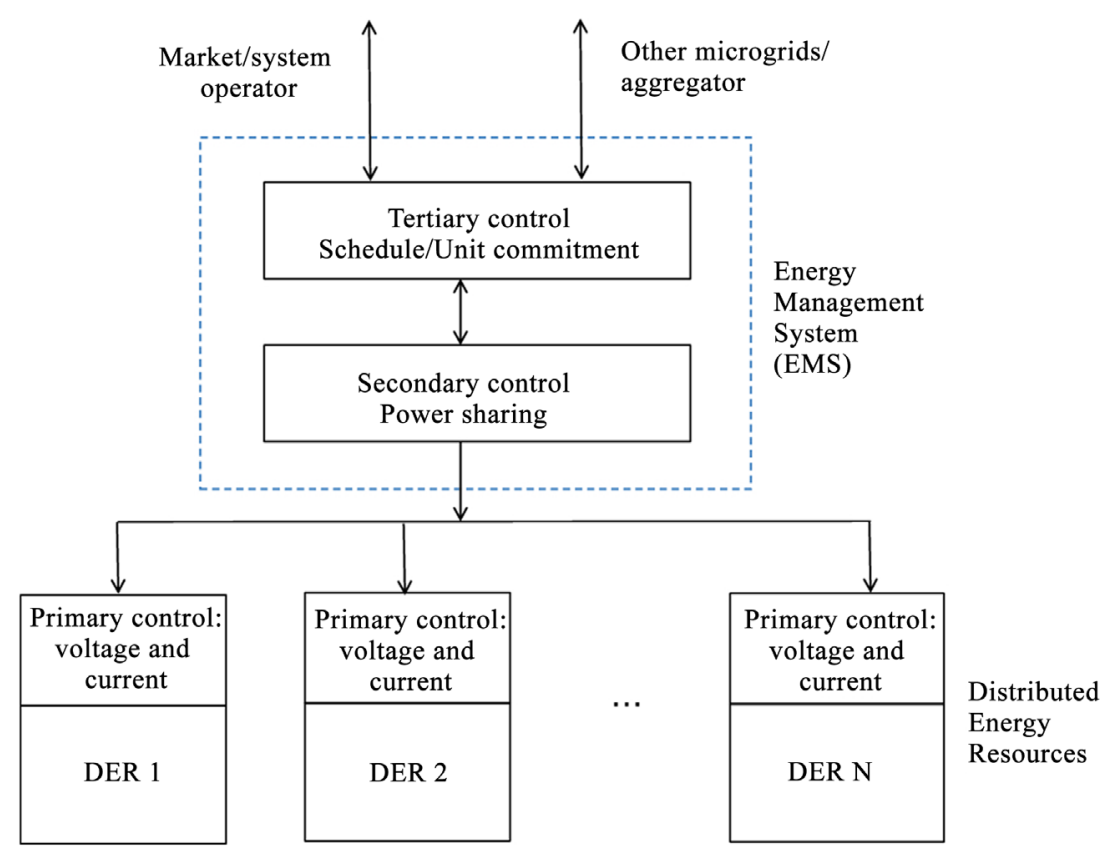

Figure 3. Microgrid operation.

\section{Control of Microgrids}

Voltage and frequency regulation, real and reactive power control, load forecasting and scheduling, microgrid monitoring, protection, and black start are all part of microgrid control. Primary voltage and frequency regulation, as well as primary real and reactive power control, are all part of the local control and protection level for each local generation and energy storage unit. Distributed generation, energy storage devices, distributional network and loads, as well as hierarchical network control and management, make up an IOT-based Microgrid. Power electronic interfaces and control strategies are utilized to integrate energy from DG to grid. Different works have shown a tendency to employ different techniques to ease network stability control utilizing IOT in this regard. A microgrid can operate in two modes: Grid-connected and Island mode. Both of these modes are requiring in different control mechanisms. Control methods can be classified into two types: Those that involve communication and those that do not. This paper is a brief overview of microgrid control techniques such as Power Quality Control, Energy Management Systems, Optimal Load Scheduling, and Multi-Objective Optimization [14].

\subsection{Power Quality Control}

The demand for higher power quality has risen as the spectrum of electronic gadgets and LEDs has expanded exponentially. Microgrids are well suited to providing greater power quality since they have the ability to regulate frequency, voltage, and cargo on a domestic level, as well as a quick-responding energy storage system. [15]. Harmonic interferences that emerge as a result of grid-to-starpanel connections are decreased by converting the system to an autonomous 
microgrid. Harmonics created in a microgrid's high-voltage supply electrical converter (VSI) largely based renewable energy supply are filtered using specifically built frequency selective filters. These frequency selective filters are made up of three parts, each of which is a space-vector quantity [16].

By constructing a DC microgrid with a cogeneration system for each house, a residential complex is provided with high-quality electricity and a constant supply. Adjusting the number of co-generation units running will affect the overall power output that is shared across residences. Super capacitors were chosen as the energy storage system in this system. In a very set of grid-interfacing system, the quality series-parallel structure is customized. The series parallel structure, for example, is used to connect the utility grid with a local grid or microgrid. These designs have been compared to traditional series-parallel systems and microgrid linked systems, demonstrating their versatility [17]. Figure 4 shows the series parallel structure applied for coupling the utility grid and a local grid/micro-grid.

Microgrids will function in both grid-connected and autonomous modes, with management variables that are fundamentally different in each mode. For the best operation of the system to work in two modes, completely separate management methods must be utilized. Two management loops are provided to a microgrid operating in autonomous mode. The PI controllers' inner loop, which is supplied with power and voltage, rejects these high-frequency disturbances and reduces the output filter, avoiding resonance with the external network. The outer loop manages droop by sharing the grid's elemental real and reactive powers with micro-sources [18]. With the goal of improving system stability, an optimization problem is designed for style controllers, filters, and power sharing coefficients. To obtain the best parameters, the particle swarm optimization (PSO) rule is used. Adapting a droop control strategy for the main interconnecting device improves facility sharing between AC and DC in a hybrid microgrid. To improve power sharing by improving droop control features, an adaptive virtual electric resistance management technique is used [19].

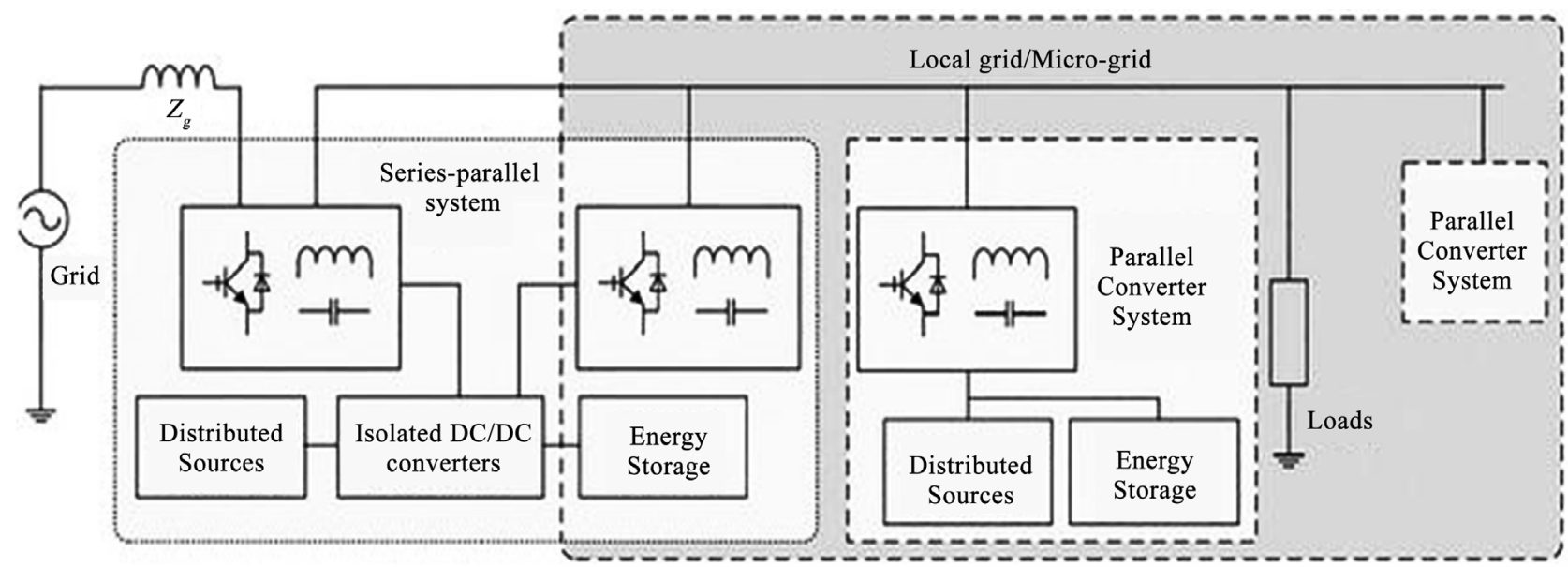

Figure 4. The series parallel structure applied for coupling the utility grid and a local grid/micro-grid. 
For dominant output, the unpredictable nature of natural resources necessitates the use of many sophisticated algorithms such as PSO and GA. A microgrid serving each AC and DC hundreds is envisioned with a dynamic management concept. Five managemental area units are used to govern power flow during this management theme and to investigate the mechanical phenomenon of reference speed. Mistreatment is used to reach a microgrid's minimum price recovery tariff. PSO is a global optimization tool. The findings of the entire search region were used as a reference for testing and validation of PSO under the condition of positive income [20].

By designing a flexible AC distribution system device, an attempt is made to optimize power quality and resiliency of the total facility to which the microgrid is attached. The device style employs a model predictive management rule that optimizes both steady state and transient state management problems separately, reducing process time [21]. The most significant downside of FACTS devices is their high cost. This is frequently overcome by misusing information-based sensible parks. To eliminate voltage imbalance and improve current limitation, a prophetic controller is used in this model. Using a decentralized management strategy, the distributed energy sources are protected against overloading. The MPC is raised using the droop approach in order to create a coordinated operation with rapid dynamic responsiveness [22].

A three-stage hierarchical control system architecture is proposed in the system. Here, the three control levels are:

1) Local micro source controllers and load controllers;

2) Microgrid system central controller;

3) Distribution management system.

Throughout transient situations, the power electronic interface offers native data to rate for the dominant voltage and frequency of the microgrid. Following the instruction of the central controller, the native MCs undertake native optimization after the MGs are connected to the facility. Active and reactive power management, as well as cargo chasing and cargo management, are all part of the native optimization. The MGCC uses market prices for energy and fuel to determine how much power a microgrid should buy from the grid, resulting in power optimization [23]. Figure 5 shows a dimension with the help of a flow chart of the multi objective Particle Swarm improvement. In the system, a branch-based power flow technique is projected that can incorporate sensible and isolated microgrid operative limitations and manage instances involving multiple DGs and droop-control operative mode. The developed methodology is derivative-free and inversion-free, making it rapid and climbable for a wide range of buses with minimal machine time and accuracy loss. The problem of determining isolated microgrids using a branch-based power supply flow has been solved by the unique forward return forward backward sweep or FR-FBS technique, which is capable of producing an influencing flow solution [24].

In a microgrid integrated with the grid, a distributed cooperative management is expected to control active power fluctuations for the Purpose of Common 


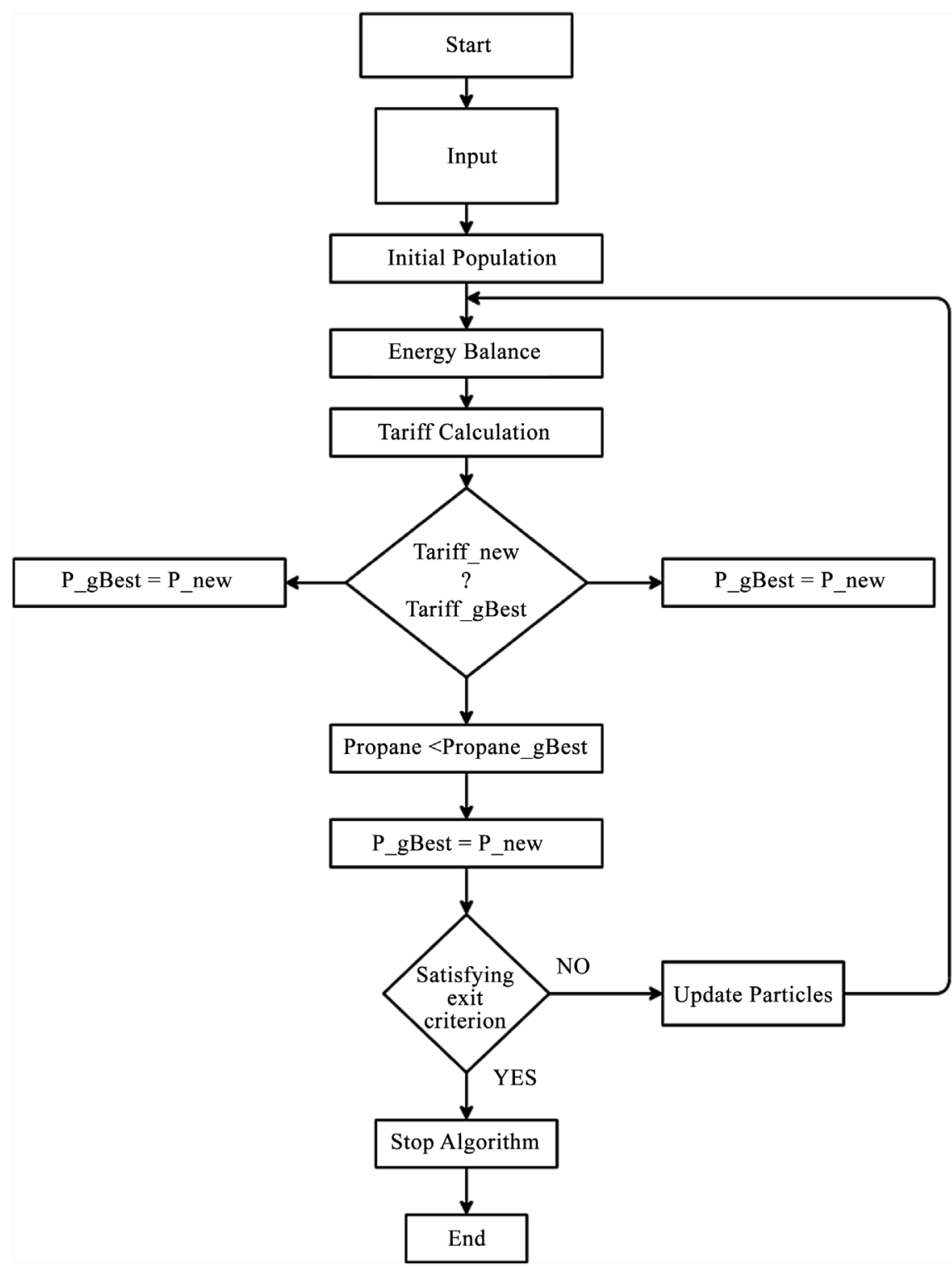

Figure 5. Flow chart of particle swarm optimization algorithms.

Coupling (PCC). By dominating the charge and discharge cycles of many energy storage units, an endeavor is produced to reduce the extra regulation strain on most grid (ESU). Every ESU's State of Charge (SoC) and quantitative Relation of Power (RoP) are shaped like a multivalent system. To keep the ESUs' SoC and RoP at the same level, a planning management rule is used. Wireless reactive power sharing with virtual resistivity enhancement improves reactive power sharing across the sources. The aim function is encased in reactive power droop slope, making it extremely adaptable to various capability systems. A distributed management technique optimizes power sharing and cargo voltage management. In order to close the generation-demand gap, ability coordination and voltage improvement occur simultaneously. For optimizing parameters of a load frequency controller, the grass-hopper improvement rule is used [25]. 


\subsection{Energy Management Systems}

The most important building component of a microgrid to reap all of its benefits is a cost-effective and optimal energy management system. For the most efficient functioning of microgrids, researchers have devised a variety of methodologies and tactics. A MG's Energy Management System (EMS) could be a sophisticated machine-controlled system largely focused on resource schedule optimization: It can optimize the management of distributed power and energy storage devices within the microgrid thanks to superior information technology. A planning problem's premise is to accurately state load and supply data; a comparative study of wind speed statements is discussed [26].

Intelligent algorithms are used to make recommendations about how devices and systems should be operated for optimal performance. It is proposed to develop an optimum generation planning model for virtual stations (VPP) that takes into account the degradation price of energy storage systems. Because of its manageable and schedulable behavior, the energy storage system is an essential component of a microgrid for flexible dispatch. Following a thorough investigation into the effects of degradation price on optimal VPP planning, it was discovered that day-ahead market rates move significantly less, and batteries with lower degradation prices are sent more frequently by the VPP. A microgrid's energy storage system's capability and size are optimized jointly. The improvement issue considers a variety of practical restrictions. The issue has been fixed piece by piece to save machine time and complexity [27].

Heuristic algorithms are ideal for energy management systems since they reduce the amount of time it takes to make a decision. On microgrids, a sophisticated heuristic formula for energy management is created. The focus of this formula is on the premise: At every opportunity, avoid wasting the current renewable potential. The management led exploitation model prognostic control topic is the reduction in output power due to device failure, shadowing of the panels, and accumulation of dirt on the PV panels. The Energy Management System'S (EMS) goal is to reduce the amount of energy drawn from the grid. The equation for the balance is as follows:

$$
P_{\text {Load }}(t)=P_{P V}(t)+P_{\text {Grid }}(t)+P_{\text {Batt }}(t)
$$

here, $P_{P V}(t), P_{\text {Batt }}(t), P_{\text {Grid }}(t)$ and $P_{\text {Load }}(t)$ are the requests from the load for PVgenerated power, given by the ESS and power grid, respectively.

The minimization problem is written as Equation from the above balance Equation (2).

$$
\sum_{t=0}^{\infty}\left\|P_{\text {Grid }}(t)\right\|_{2}^{2}=\sum_{t=0}^{\infty}\left\|P_{\text {Load }}(t)-P_{P V}(t)-P_{\text {Batt }}(t)\right\|_{2}^{2}
$$

A probabilistic model using a revised scenario-based decision-making methodology is used to achieve optimal day-ahead programming of electrical and thermal energy resources. The demand response-aware concurrent energy and reserve programming paradigm has been mentioned. Modeling uncertainty in operational problem solving makes the planned outcomes more realistic. By in- 
stalling an intelligent energy management system that supports close to correct generating power interpretation and optimum power flow, costs are successfully minimized [28].

The storage states and frequency of renewable energy systems were taken into account using an intelligent technique. In addition, a distributed Intelligent Energy Management System is used to reduce the operational costs of a microgrid based on a model electrical phenomenon. The Fuzzy Adjustive Resonance Theory (ART) map neural network is used to construct a heuristics-based forecast of PV generation as part of the improvement theme. To mimic the unpredictable character of a microgrid, affine arithmetic is used. The random weight trade-off particle swarm improvement algorithmic software lowers the price. To meet operational limits and unpredictable energy demand, the best energy management approach for energy storage is created as a Mixed-Integer Linear Improvement downside (MILP) [29]. The primary goal is to reduce the system's price function, while the secondary goals are to meet customer demand and ensure safety. Before discussing improvement, there is a storage strategy in which the energy state of the storage system is pre-determined, and another storage strategy in which the storage energy state is defined when a multi-objective improvement problem gives a set of answers. A competent in nursing must select one of them based on his experience and background. The design and operation of a microgrid are multi-objective improvements that have been optimized. By using fuzzy satisfaction-maximizing methods, the multi-objective improvement problem is reborn as a single improvement problem. The resurrected disadvantage is victimization MILP [30].

For power mercantilism, an attempt is made to model associate in nursing energy mercantilism system. The model has two stages: The planner and the short-time equalization mechanism. The Planner envisions the programming of many predefined activities in order to achieve partial demand aspect management and obtain statistics on ability usage. The Planner also calculates background usage and projects the ability to produce energy from renewable sources. In a Short-Time Equalization System, a multiagent, distributed system manages governable sources to achieve power balance in a short period of time. When the energy management problem of two cooperative microgrids is addressed, a new idea of energy cooperation is planned. The improvement is initially obtained via an off-line manner. Associate in nursing on-line improvement methodology is produced to support the solutions obtained for energy collaboration [31].

Figure 6 shows the general structure of two-stage optimization system.

For the economic operation of an islanded community microgrid, a graded coordination method with primary, secondary, associate degreed tertiary coordination is envisioned. The coordination technique is designed to allow for flexible and optimal power exchange coordination while maintaining the typical operation of participating microgrids. The inter-linking converter droop has been modified and applied to communal microgrid coordination. Implementing 


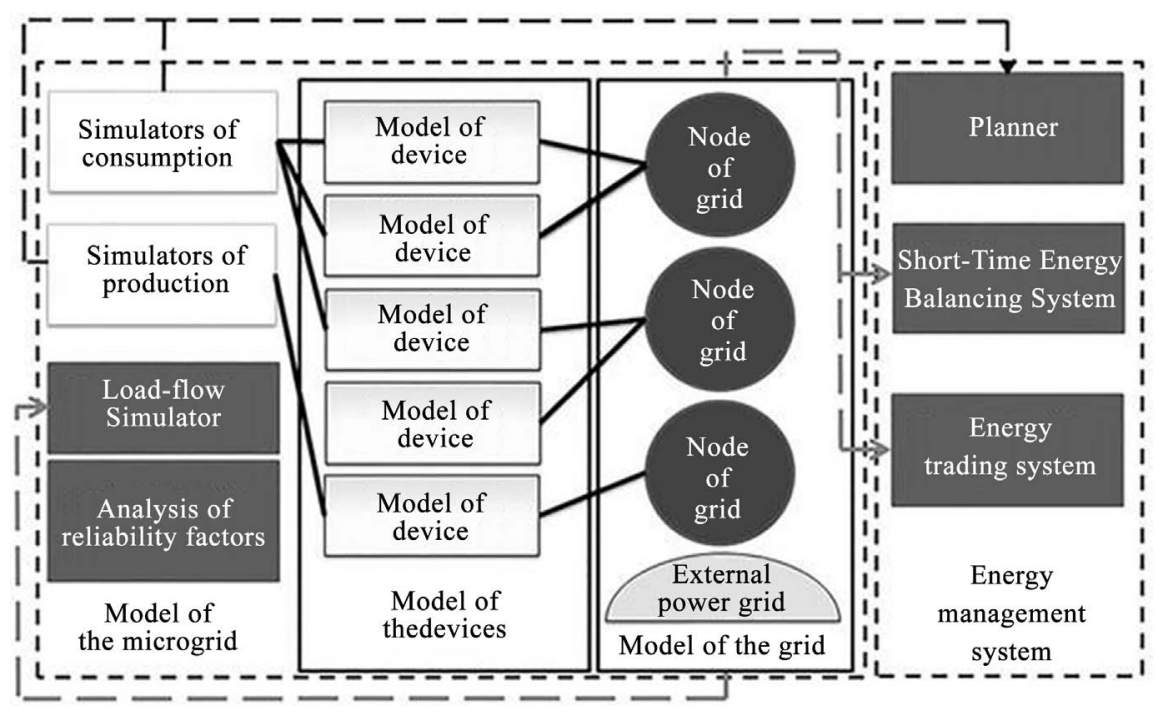

Figure 6. The general structure of two stage optimization system.

a Radial Basis Perform Neural Network (RBFNN) combining particle swarm optimization by dominant each active and reactive power of battery energy storage system on-line improves the stability of a complete microgrid [32].

The development of a novel management algorithmic program for demand response management and thermal comfort optimization in microgrids made up of a PV array, a turbine, and an energy storage unit. The planned study intends to create a scalable and effective demand response program that can integrate the goal of balancing energy generation and consumption with resident behavior [33]. Within the designed algorithmic program, a two-level higher-up method is used. A lower-level control is provided by an area managemental at each generating unit, and a higher-level control is provided by a centralized managemental. The operational programming drawback was created in accordance with a variety of incentives. Such as Peak Time Rebate (PTR) and Demand Response (DR) programming for responsive masses, as well as limits, such as optimum battery and diesel generator programming [34].

By modeling the operational designing downside as a MILP, the major goal is to reduce the value of a microgrid. Unit linearized exploitation constant realizations are used to model supply and cargo uncertainty. The goal is to create a microgrid central energy management system that is based on day-ahead operational planning and a web adjustment mechanism that runs throughout the operation. Unit Commitment (UC) is a degree optimization problem that is used to confirm the operating schedule of producing units at each hour interval with varying masses under various limitations and environments [35]. A revised Genetic Algorithmic program (GA) based technique is intended to solve the unit commitment problem with a multi-objective perform in order to reduce the economic value and $\mathrm{CO}_{2}$ equivalent emissions utilizing a dynamic programming-based algorithmic program. To speed up the convergence of a genetic algorithmic program, a simulated tempering technique is used [36]. 
Microgrids will benefit from a Multi-Agent System (MAS) technology that provides optimal operation approaches. It is suggested that the repair and management of microgrid exploitation MAS. To optimal multi microgrid operation, a ranked power and energy management system is supported. In the most recent upgrade, a multi-agent system was created. The agent's communication is governed via a Modified Contract Web Protocol (MCNP). MILP (mixed number applied math) models were created for each step [37]. The distributed energy management of a microgrid with two-star generators is optimized using a Multi-Agent System technique. The Java Agent Development Environment (JADE) is used to create a MAS model that optimizes power generation under the unpredictable nature of the star PV system and cargo. Using this approach, a client can examine all logical sequences of options and choose the best energy management actions to increase operational efficiency in a highly distributed environment. The invention is a multi-layer Emmet colony optimization-based energy management system for a microgrid. The main goal is to reduce the value of ability generation by using hourly day-ahead and real-time programming. In an excessively native energy market (LEM) system, this algorithmic program aims to supply the load demand with the least amount of energy [38].

\subsection{Optimal Load Scheduling}

When it comes to load control, once demand is low, responsive hundreds are managed. The random character of load must be considered in order to achieve optimal load control. A random model for microgrid short-term planning is being developed. With the planned variable process module, this model takes into account the uncertain nature of call variables (SVCM). The Monte-Carlo simulation, which is more commonly used in the SVCM algorithmic program, gives radically different outcomes. This approach has a substantial impact on energy and reserve allocation for units, as well as compulsory load curtailment. It is designed a random programming improvement model with risk neutral and risk antipathetic options. A genetic algorithmic program (GA)-based planning to lower each day-ahead and real-time adjustment prices are supported by a two-point estimate-based probabilistic period optimum power flow. The rolling horizon Andrei Markov call method is used to solve the planning drawback (MDP). Victimization greedy algorithmic program is a proposed basic policy [39].

A multi-period artificial bee colony improvement algorithmic program is used to answer the problem of cost-effective dispatch of responsive hundreds. To estimate non-dispatch ready electricity generation and freight demand while accounting for uncertainties, a man-made neural network paired with a Markoff chain (ANN-MC) technique is used. In order to stimulate the use of renewable energy sources and to ensure a high level of charge inside the storage system for the long term, the planning is improved by include penalties. Linear models are intended for use in planning that is based on pure mathematics in general (GAMS). A Microgrid Energy Commercialism Model (METM) lowers the overall energy purchase price by coordinating power prediction and day-ahead 
commercialism to encourage correct day-ahead commercialism electrical demand [40].

Excessive loading or generation occurs while using the Islanding method because the ability exchange for the purpose of common coupling is forced to zero. The Probability of Booming Islanding (PSI) has been created. When a microgrid is flash islanding from the most grid, PSI evaluates the possibility of the microgrid keeping enough spinning reserve (both up and down) to meet native demand and accommodate native renewable energy. To optimize material demands of the Islanded Microgrid with electrical phenomena and Energy storage devices, the time sequent simulation methodology is used (IMPE) [41].

A problem with bi-level improvement has been fixed. The higher-level goal of the intended model is to increase the distribution company's profit, while the lower-level goal is to reduce the price of the microgrid. Another interactive operating method is envisaged for the microgrid in conjunction with a distributed system-supported demand response. Using sensitivity analysis, a relationship between microgrid interface capability and interaction price is discovered. The uncertainties of renewable sources and cargo demands are factored into the Unit Commitment (UC) and Economic Dispatch (ED) issues using a probabilistic forced approach. Victimization random dynamic programming is used to resolve the UC. Considering the probabilistic behaviors to solve the optimal power dispatch drawback. The capacity generated by each source, as well as the load associated with each supply, is depicted using a probability distribution diagram (PDF). When getting PDFs of random variables using an imperialist competitive algorithmic program, the ability dispatch problem is solved (ICA) [42].

When integrating large populations of heat pumps and electric vehicles into a traditional power dispatch system, a stratified load management approach is used. The suggested methodology boosts the capability system's technical and financial performance. To accommodate enormous load changes, the building thermal mass is combined with simple control systems. A self-activating air-source configuration (HP) athletics controls load fluctuations. Adapting bus level management improves the capabilities and voltage ramping rates. It is planned to provide a generalized formulation to determine the best operational strategy and value improvement theme for a Microgrid (MG) for residential use. A nonlinear forced multi-objective improvement drawback is used to create the planned drawback [43].

\subsection{Multi-Objective Optimization}

Multi-objective optimization is a branch of multiple basis decision that deals with optimization problems involving one or more objective functions that must be optimized simultaneously. The target functions and restrictions in a microgrid power management downside are non-linear in nature. Furthermore, these objective functions are incompatible and contradictory in several ways. It becomes necessary to introduce economic expert optimality at this point. When 
multiple objective functions are optimized at the same time, the result may not be optimal but it cannot be improved without deteriorating the opposite. This is referred to as an economic expert optimum response. The decision maker has the option of selecting an answer that is a lot more acceptable in terms of the preferences [44].

Opening is to define the preferences for designing a multi-objective optimization downside for a microgrid. The preferences include a number of goals that must be accomplished for proper grid operation, as well as a few non-traditional operations. These preferences are based on the buyer's relative importance [45].

A collection of intelligent methods is for incorporating a variety of strategies such as heuristic and biological process algorithms. They will handle many objectives at the same time, resulting in a shorter procedure time and a more accurate result. On a twenty-four-hourtime, horizon an optimization model for a microgrid operational system in each grid-connected and autonomous mode has been planned. The settled model and the random model are offered as optimization models. According to the predicted power generation from renewable energy sources, the finalized model lowers the cost of operating a microgrid. The random optimization model takes into consideration the randomness in renewable energy sources' power output while supporting prescribed scenarios. It is mentioned that a multi-objective and multi-constraint optimization process is used. As the demand for reliable and faster solutions to the power management problem grows, computationally intelligent approaches are being deployed. A multi-objective random optimal designing technique and a random chanceconstrained programming model are offered to achieve economic and environmental optimization for a complete microgrid. PEVs (plug-in electric cars) are seen as a haphazard issue. The matter's aim functions are to maximize the voltage security margin while minimizing total power losses, total current costs, and total emissions from power sources [46]. Figure 7 shows the flow chart of the multi-objective particle swarm optimization.

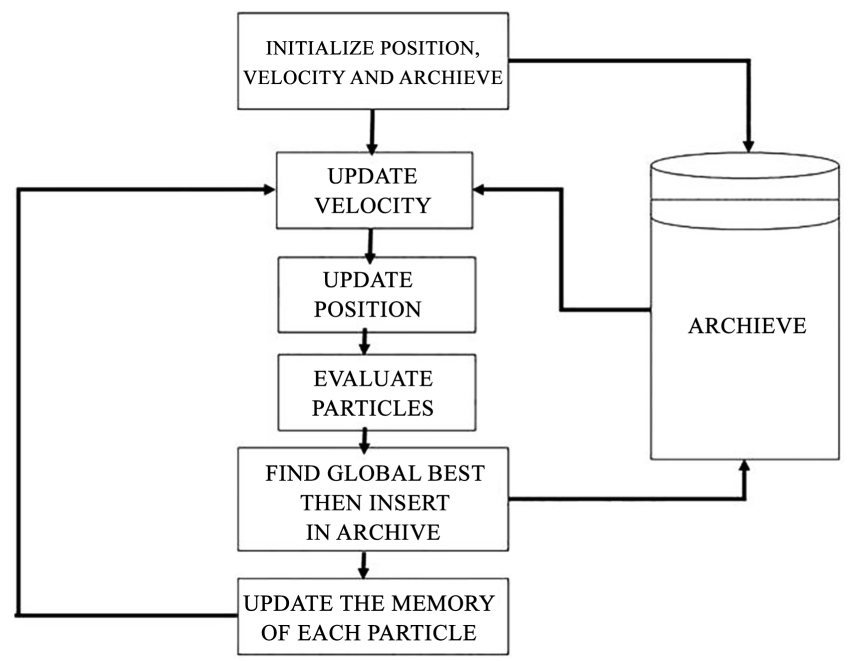

Figure 7. Flow chart of the multi-objective particle swarm optimization. 
Multiple random repetitions of the MOPSO on a fairly large set of Pareto-optimal trade-off solutions are frequently produced here. To handle the proposed integrated renewable energy theme, a self-tuned PID interface was used. The results of the improvement demonstrated that multi objective Particle Swarm improvement is extremely effective in managing power transfer from renewable sources to various DC masses in autonomous mode and to the grid in linked mode, and that it can also retain the best power quality.

To solve the energy-saving power generation dispatch problem, a multi-agent system is used in conjunction with cooperative game theory. The BDI bus agents are built as players and a distributed tree network as the cooperative structure. The author speaks resolution for close to inexpensive dispatching of generated power to the tree arranged power network provides a sociology best operative purpose. In a microgrid system, multi-criteria call analysis-based technique is used to program the dispatch. To disengage the multi-objective dispatch helpful, goal acquisition programming is used and distinctive compromise programming is used as the MCDA technique for rating the dispatch options every hour for the decision maker. Levy-Harmony has proposed to deal with various objectives using a bio-inspired improvement rule. To compute the most effective and logical worth of objectives, a triangle accumulation model was designed. It was possible to reach a well-balanced accuracy and search rate square standard [47].

\section{Discussion and Critical Thinking}

Following a survey of microgrid architectures, it was discovered that the vast majority of testbeds are AC microgrids. The AC microgrid is straightforward to link with the grid because the grid and most hundreds are AC. One of the most important responsibilities in $\mathrm{AC}$ systems is to maintain the ability quality. On the other hand, the most significant advantage of DC systems is that they have fewer power quality concerns, requiring less extra administration and components. However, because of a lack of sufficient DC hundreds, the usage of a DC microgrid is severely limited. The HFAC microgrid may be a novel concept, but it might be a viable way to integrate renewable energy sources into the microgrid. One of the most advantageous aspects of this approach is that PQ problems are reduced. The most significant drawbacks of the HFAC microgrid system are the complexity of the management devices, large fallout and higher long-distance power loss, which now restrict its practical application but may be investigated further. The Star PV, wind, micro-hydro, and diesel are the most often utilized decigram sources in microgrid systems. Apart from conventional sources, RES is quite popular as decigram source in European areas. In a microgrid system, power quality might be a concern. Variability of the resource creates some PQ problems since renewable decigram sources are very reliant on setting. As a result, considering PQ performance for any microgrid system is critical. Few microgrid test-beds include mandated power quality devices, according to a review of the test-beds. As a result, more research is needed to improve their PQ and 
resiliency, and therefore improve the performance of microgrid systems [48].

One of the crucial options that a microgrid should have for its self-contained and steady functioning is a storage system. The majority of current test-beds feature battery storage, however some also incorporate capacitance banks and flywheels. A number of the microgrids contain a combination of two or three storage units, while others have none at all. According to the evaluation, if there is no device, a minimum of one tolerable decigram supply is given to the system in most situations (except two). If the microgrid system has no storage devices and relies exclusively on renewable energy as a source of power, grid integration may be a good option. More research might be done in this area. Despite the fact that the microgrid has made significant progress in operational management, energy management, and conservation technologies over the last 10 years, difficulties such as small inertia, nonlinear load size relation growing, and damage ionic harm continue to limit its use. Factors that influence the microgrid's ability to operate reliably include:

1) A microgrid might be a high-capacity power supply system in and of itself. It has a limited power capability that is similar to the load. Unlike the various types of masses which have a wide range of dynamic variations, the bus voltage can change in a wide range of ways. In order to maintain stability, the energy storage instrumentation must be forced to respond quickly. Power supply and cargo matching are critical requirements for facility operation especially in microgrids. The average power hypothesis, on the other hand, is backed by ancient load applied math methods. Nonlinear masses that introduce harmonics into the microgrid are exempt from these rules. Furthermore, this theory disregards the impact of harmonics on load and power supply. For example, when a power supply system is built in harmonics, the active power output of a diesel generator with a rated power of 120 kilowatts is barely fifty-five kilowatts. The action principle of the harmonic and the electric motor is not well understood. Power matching has been a tangle in the engineering application, with the answer being to tack together greater power motors, resulting in resource waste under the circumstance of huge harmonics. The common modules of converters and systems are now supported by the research methods. The common model on the other hand, is unable to properly characterize the time-variant feature of converters. The rapid power theory presents a potential approach to handle time-variant and nonlinear problems in accordance with the analytical need of nonlinear wattage systems. The students have used the rapid power theory to develop a large number of nonlinear facility investigations both at home and overseas. Power electronics technology is used in distributed generating. The voltage flicker and the introduction of a high number of harmonics have a significant influence on power quality. Natural circumstances impact a large portion of distributed power making it difficult to create steady electricity. The interaction between distributed units and medium voltage feedback devices, as well as large variations in output, can create voltage flicker and have an influence on the power grid. Voltage fluctuation problems such as voltage pulse, voltage 
flicker and power supply interruption can be solved by improving the dependability of distributed generation and using energy storage equipment. It encourages the advancement of energy storage devices in terms of energy density, reaction time, and cost performance.

2) When power electronic devices are used in a microgrid, the issue of device cascade stability arises. Its resistivity is time-varying and nonlinear, as it is in nonlinear shift circuits. When the structure of the facility's electronic equipment is complicated, typical resistivity quantitative relation techniques are difficult to forecast the soundness. Adding a go-between filter and a planning bus controller, in addition to modifying the resistivity quantitative relationship, are useful techniques for improving cascade stability [49].

These solutions turn excellent microgrids with remote management capabilities into IoT-enabled microgrids. These include DERs such as photovoltaics, wind turbines, combined heat and power, fuel cells, and energy storage systems, as well as controllable masses such as heating, ventilation, and air-conditioning systems and electric cars. In order to do their work, active grid management systems need information from all throughout the grid. This is where the value of IoT technology comes in making it simpler to acquire and analyze data from old grid resources, DERs, and alternative electrical assets, allowing utilities to take remedial action more rapidly once connected. With this information, IoT-driven grid management systems will facilitate operations perform important functions including:

1) Optimizing line voltage to reduce the energy losses and source line damage;

2) Locating the supply of sags, surges and outages;

3) For upload leveling and restoring services quicker and creating safer override decisions;

4) Characteristic the supply of technical and nontechnical losses within the system, reducing the prices of service;

5) Lowering outage investigation times by uninflected fault locations.

With the rapid growth of renewable energy resources in microgrids, their protection and command may be handled with the help of IOT. This continuous operation and automation of the system allow for energy optimization and monitoring of the system's status. The Internet of Things allows the system to monitor and supervise the network from afar. IOT was used in microgrids in this article to monitor and protect the network in both grid-connected and islanded modes of operation. Furthermore, the energy pattern provided by the supply switch was complete because mainly based functioning is prioritized, extra safe and continuous supply is guaranteed with the addition of additional sources and masses inside the system. This implementation on a bench demonstrates its potential for microgrid automation and energy improvement [50].

Table 1 shows the comparison between other papers and our paper, though our paper has the same purpose to accomplish the task. Table 2 shows the overall comparison between our paper and other systems of the same system. The 
Table 1. Comparison with other papers.

\begin{tabular}{ccccc}
\hline No. & Name & Energy Source & Storage & $\begin{array}{c}\text { The connection between } \\
\text { microgrid and control device }\end{array}$ \\
\hline 1 & This Paper & Non-renewable & Battery & wireless \\
2 & Ref [12] & Renewable & Super Capacitor & wireless \\
3 & Ref [17] & Renewable & Battery & wireless \\
4 & Ref [19] & Non-renewable & Battery & wireless \\
5 & Ref [23] & Renewable & Flywheel & wireless \\
6 & Ref [25] & Non-renewable & Super Capacitor & wireless \\
\hline
\end{tabular}

Table 2. Overall comparison.

\begin{tabular}{cccccc}
\hline No. & Name & Communication & Control technology & Modification & Operation \\
\hline 1 & This Paper & Two-way & Robust \& wireless & Possible & Digital \\
2 & Ref [12] & Two-way & Robust & Possible & Digital \\
3 & Ref [17] & Two-way & Robust & Possible & Digital \\
4 & Ref [19] & Sensor based & Robust \& wireless & Possible & Digital \\
5 & Ref [23] & Multi-way & Robust & Possible & $\begin{array}{c}\text { Digital \& } \\
\text { self-monitoring }\end{array}$ \\
& & & & & Pigital \& \\
6 & Ref [25] & Two-way & Robust & Possible & self-monitoring \\
\hline
\end{tabular}

significance of this comparison is to focus on the advantages and flexibilities for the advance use of IoT in microgrids.

For the same sort of energy source, this paper is similar to [19] [25]. Here, the energy source is non-renewable. Because nonrenewable energy is less expensive than renewable energy, we choose to use it. [12] [17] [23] make use of a somewhat expensive renewable energy source. It has a comparable storage method to this article, [17] [19], which also employ batteries for storage. The use of a battery for storage is ideal for this method. Whereas [12] [25] make use of a super capacitor, which is both costly and complicated. [23] Makes use of a flywheel. The connection mechanism between the micro grid and the IoT compatible device in this article, [12] [17] [19] [23] [25], is the same; the connection method is wireless.

In this paper [12] [17] [25], the communication mechanism is two-way. Although this is the usual method for almost $90 \%$ of systems, several others employ a multi-way communication mechanism. This paper and [19] apply the same control technology. This technology has been refined to the point that consumers can readily grasp the system. In this location, the occurrence of malfunctioning has been reduced. Modifications are also available for all of the papers listed in Table 2. Users might change the system in some way to enhance flexibility. This paper, [12] [17] [19] operating processes are all digital. For the digital and self-monitoring processes [23] [25]. It is a fairly frequent procedure 
all across the world, but making it self-monitoring adds the benefit of time savings. These comparison scenarios demonstrate that we need to make certain improvements in the near future to make the IoT-based micro grid system more efficient. Furthermore, our tested system may not be up to par.

\section{Conclusion}

An expanded outcome for IoT-based microgrids will aid us in the use of electronic power energy in the near future. Microgrids with IoT will distribute renewable energy resources in each grid in a way that is both compatible and comprehensive. Many different improvement techniques and intelligent improvement algorithms will be required in the area unit, each with its own set of benefits and drawbacks. If we can use IoT to operate microgrids, the adaptability will be greatly improved. In the near future, the procedure will require more improvements. Because this procedure is entirely reliant on wireless commands, the cost of connecting gear will be significantly reduced. Microgrids will also be more appealing to people all around the world.

\section{Acknowledgements}

Avoid the stilted expression, “One of us (R. B. G.) thanks...” Instead, try “R. B. G. thanks". Do NOT put sponsor acknowledgements in the unnumbered footnote on the first page, but here. Authors would like to thank the Department of Electrical and Computer Engineering of North South University.

\section{Conflicts of Interest}

The authors declare that they have no conflicts of interest to report regarding the present study.

\section{References}

[1] Fadel, A.M., Ibrahim, A.M., Tamazin, A.K., Hamdy, R.A. and Abdel-Khalik, A.S. (2019) IoT-Based Power Management for DC Microgrids. 201921 st International Middle East Power Systems Conference (MEPCON), Cairo, 17-19 December 2019, 1107-1111. https://doi.org/10.1109/MEPCON47431.2019.9008216

[2] Bifano, W.J., Ratajczak, A.F., Bahr, D.M. and Garrett, B.G. (1979) Social and Economic Impact of Solar Electricity at Schuchuli Village. Solar Technology in Rural Settings: Assessments of Field Experiences.

[3] Hina Fathima, A. and Palanisamy, K. (2015) Optimization in Microgrids with Hybrid Energy Systems: A Review. Renewable and Sustainable Energy Reviews, 45, 431-446. https://doi.org/10.1016/j.rser.2015.01.059

[4] Cobben, J.F.G., Kling, W.L. and Myrzik, J.M.A. (2005) Power Quality Aspects of a Future Micro Grid. International Conference on Future Power Systems, Amsterdam, 16-18 November 2005, 1-5. https://doi.org/10.1109/FPS.2005.204282

[5] Illindala, M. and Venkataramanan, G. (2012) Frequency/Sequence Selective Filters for Power Quality Improvement in a Microgrid. IEEE Transactions on Smart Grid, 3, 2039-2047. https://doi.org/10.1109/TSG.2012.2197834

[6] Kakigano, H., Miura, Y. and Ise, T. (2010) Low-Voltage Bipolar-Type DC Microgrid 
for Super High-Quality Distribution. IEEE Transactions on Power Electronics, 25, 3066-3075. https://doi.org/10.1109/TPEL.2010.2077682

[7] Wang, F., Duarte, J.L. and Hendrix, M.A.M. (2011) Grid-Interfacing Converter Systems with Enhanced Voltage Quality for Microgrid Application Concept and Implementation. IEEE Transactions on Power Electronics, 26, 3501-3513. https://doi.org/10.1109/TPEL.2011.2147334

[8] Hassan, M.A. and Abido, M.A. (2011) Optimal Design of Microgrids in Autonomous and Grid-Connected Modes Using Particle Swarm Optimization. IEEE Transactions on Power Electronics, 26, 755-769.

https://doi.org/10.1109/TPEL.2010.2100101

[9] Manju, S., Seema, P.N. and Rajendran, A. (2016) A Novel Algorithm for Power Flow Management in Combined AC/DC Microgrid. 1st IEEE International Conference on Power Electronics, Intelligent Control and Energy Systems (ICPEICES2016), Delhi, 4-6 July 2016, 1-6. https://doi.org/10.1109/ICPEICES.2016.7853428

[10] Shaban, M. and Ben Dhaou, I. (2021) Design of an IoT-Enabled Microgrid Architecture for a Partial Grid-Connected Mode. 2021 18th International Multi-Conference on Systems, Signals \& Devices (SSD), Monastir, 22-25 March 2021, 1115-1119. https://doi.org/10.1109/SSD52085.2021.9429408

[11] Adel, M., Sharaf, A. and El-Gammal, A.A. (2010) A Novel Efficient PSO-SelfRegulating PID Controller for Hybrid PV-FC-Diesel-Battery Micro Grid Scheme for Village/Resort Electricity Utilization. IEEE Electrical Power \& Energy Conference, Halifax, 25-27 August 2010, 1-6.

[12] Sigarchian, S.G., Orosz, M.S., Hemond, H.F. and Malmquist, A. (2016) Optimum Design of a Hybrid PVCSPLPG Microgrid with Particle Swarm Optimization Technique. Applied Thermal Engineering, 109, 1031-1036. https://doi.org/10.1016/j.applthermaleng.2016.05.119

[13] Tan, K.T., So, P.L., Chu, Y.C. and Chen, M.Z.Q. (2013) A Flexible AC Distribution System Device for a Microgrid. IEEE Transactions on Energy Conversion, 28, 601-610. https://doi.org/10.1109/TEC.2013.2260162

[14] Divya, R., Nair, D.S., Nair, M.G. and Ilango, K. (2016) Tariff Based Fuzzy Logic Controller for Active Power Sharing between Microgrid to Grid with Improved Power Quality. 2016 International Conference on Energy Efficient Technologies for Sustainability (ICEETS), Nagercoil, 7-8 April 2016, 406-409.

[15] Golsorkhi, M.S. and Lu, D.D.-C. (2016) A Decentralized Control Method for Islanded Microgrids under Unbalanced Conditions. IEEE Transactions on Power Delivery, 31, 1112-1121. https://doi.org/10.1109/TPWRD.2015.2453251

[16] Tiwari, V., Kumari, S. and Sahoo, P.P. (2021) Monitoring and Control of PV Microgrid Using IoT. 20217 th International Conference on Electrical Energy Systems (ICEES), Chennai, 11-13 February 2021, 292-298. https://doi.org/10.1109/ICEES51510.2021.9383724

[17] Nassar, M.E. and Salama, M.M.A. (2017) A Novel Branch-Based Power Flow Algorithm for Islanded AC Microgrids. Electric Power Systems Research, 146, 51-62. https://doi.org/10.1016/j.epsr.2017.01.019

[18] Huang, C., Weng, S., Yue, D., Deng, S., Xie, J. and Ge, H. (2017) Distributed Cooperative Control of Energy Storage Units in Microgrid Based on Multi-Agent Consensus Method. Electric Power Systems Research, 147, 213-223. https://doi.org/10.1016/j.epsr.2017.02.029

[19] Zhu, Y., Fan, Q., Liu, B. and Wang, T. (2018) An Enhanced Virtual Impedance Op- 
timization Method for Reactive Power Sharing in Microgrids. IEEE Transactions on Power Electronics, 33, 10390-10402. https://doi.org/10.1109/TPEL.2018.2810249

[20] Barik, A.K. and Das, D.C. (2018) Expeditious Frequency Control of Solar Photovoltaic/Biogas/Biodiesel Generator Based Isolated Renewable Microgrid Using Grasshopper Optimization Algorithm. IET Renewable Power Generation, 12, 1659-1667. https://doi.org/10.1049/iet-rpg.2018.5196

[21] Moayedi, S. and Davoudi, A. (2017) Unifying Distributed Dynamic Optimization and Control of Islanded DC Microgrids. IEEE Transactions on Power Electronics, 32, 2329-2346. https://doi.org/10.1109/TPEL.2016.2565517

[22] Delfino, F., Rossi, M., Pampararo, F. and Barillari, L. (2016) An Energy Management Platform for Smart Microgrids. In: Intelligent Computing Systems, Springer, Berlin, 207-225. https://doi.org/10.1007/978-3-662-49179-9_10

[23] Kiranvishnu, K. and Sireesha, K. (2016) Comparative Study of Wind Speed Forecasting Techniques. 2016 Biennial International Conference on Power and Energy Systems. Towards Sustainable Energy (PESTSE), Bangalore, 21-23 January 2016, $1-6$.

[24] Zhou, B., Liu, X., Cao, Y., Li, C., Chung, C.Y. and Chan, K.W. (2016) Optimal Scheduling of Virtual Power Plant with Battery Degradation Cost. IET Generation, Transmission \& Distribution, 10, 712-725. https://doi.org/10.1049/iet-gtd.2015.0103

[25] Akram, U., Khalid, M. and Shafiq, S. (2017) An Innovative Hybrid Wind-Solar and Battery-Supercapacitor Microgrid System-Development and Optimization. IEEE Access, 5, 25897-25912. https://doi.org/10.1109/ACCESS.2017.2767618

[26] Tomoiaga, B., Chindris, M.D., Sumper, A. and Marzband, M. (2013) The Optimization of Microgrids Operation through a Heuristic Energy Management Algorithm. Advanced Engineering Forum, 8-9, 185-194. https://doi.org/10.4028/www.scientific.net/AEF.8-9.185

[27] Riar, B., Lee, J., Tosi, A., Duncan, S., Osborne, M. and Howey, D. (2016) Energy Management of a Microgrid: Compensating for the Difference between the Real and Predicted Output Power of Photovoltaics. 2016 IEEE 7 th International Symposium on Power Electronics for Distributed Generation Systems (PEDG), Vancouver, 27-30 June 2016, 1-7.

[28] Zamani, A.G., Zakariazadeh, A., Jadid, S. and Kazemi, A. (2016) Stochastic Operational Scheduling of Distributed Energy Resources in a Large-Scale Virtual Power Plant. International Journal of Electrical Power and Energy Systems, 82, 608-620. https://doi.org/10.1016/j.ijepes.2016.04.024

[29] Chakraborty, S. and Simoes, M.G. (2008) PV-Microgrid Operational Cost Minimization by Neural Forecasting and Heuristic Optimization. Industry Applications Society Annual Meeting, Edmonton, 5-9 October 2008, 1-8.

https://doi.org/10.1109/08IAS.2008.147

[30] Mohan, V., Suresh, R., Singh, J.G., Ongsakul, W. and Madhu, N. (2017) Microgrid Energy Management Combining Sensitivities, Interval and Probabilistic Uncertainties of Renewable Generation and Loads. IEEE Journal on Emerging and Selected Topics in Circuits and Systems, 7, 262-270. https://doi.org/10.1109/JETCAS.2017.2679030

[31] De Leone, R., Giovannelli, A. and Pietrini, M. (2016) Optimization of Power Production and Costs in Microgrids. Springer-Verlag, Berlin.

[32] Chen, J., et al. (2018) Optimal Sizing for Grid-Tied Microgrids with Consideration of Joint Optimization of Planning and Operation. IEEE Transactions on Sustainable Energy, 9, 237-248. https://doi.org/10.1109/TSTE.2017.2724583 
[33] Radziszewska, W. and Nahorski, Z. (2016) Microgrids and Management of Power. In: Challenging Problems and Solutions in Intelligent Systems, Springer, Cham, 161-178. https://doi.org/10.1007/978-3-319-30165-5_8

[34] Kondoro, A., Dhaou, I.B. and Tenhunen, H. (2020) Enhancing the Security of IoT-Enabled DC Microgrid Using Secure-MQTT. 2020 6th IEEE International Energy Conference (ENERGYCon), Tunis, 28 September-1 October 2020, 29-33. https://doi.org/10.1109/ENERGYCon48941.2020.9236448

[35] Rahbar, K., Chai, C.C. and Zhang, R. (2018) Energy Cooperation Optimization in Microgrids with Renewable Energy Integration. IEEE Transactions on Smart Grid, 9, 1482-1493. https://doi.org/10.1109/TSG.2016.2600863

[36] Kerdphol, T., Qudaih, Y., Watanabe, M. and Mitani, Y. (2016) RBF Neural Network-Based Online Intelligent Management of a Battery Energy Storage System for Stand-Alone Microgrids. Energy, Sustainability and Society, 6, 5.

https://doi.org/10.1186/s13705-016-0071-2

[37] Korkas, C.D., Baldi, S., Michailidis, I. and Kosmatopoulos, E.B. (2016) Occupancy-Based Demand Response and Thermal Comfort Optimization in Microgrids with Renewable Energy Sources and Energy Storage. Applied Energy, 163, 93-104. https://doi.org/10.1016/j.apenergy.2015.10.140

[38] Shen, J., Jiang, C., Liu, Y. and Qian, J. (2016) A Microgrid Energy Management System with Demand Response for Providing Grid Peak Shaving. Electric Power Components and Systems, 44, 843-852. https://doi.org/10.1080/15325008.2016.1138344

[39] Umeozor, E.C. and Trifkovic, M. (2016) Operational Scheduling of Microgrids via Parametric Programming. Applied Energy, 180, 672-681.

https://doi.org/10.1016/j.apenergy.2016.08.009

[40] Kanchev, H., Colas, F., Lazarov, V. and Francois, B. (2014) Emission Reduction and Economical Optimization of an Urban Microgrid Operation Including Dispatched PV-Based Active Generators. IEEE Transactions on Sustainable Energy, 5, 1397-1405. https://doi.org/10.1109/TSTE.2014.2331712

[41] Saravanan, B., Siddharth, D., Surbhi, S. and Kothari, D.P. (2013) A Solution to the Unit Commitment Problem-A Review. Frontiers in Energy, 7, 223-236.

https://doi.org/10.1007/s11708-013-0240-3

[42] Liang, H.Z. and Gooi, H.B. (2010) Unit Commitment in Microgrids by Improved Genetic Algorithm. 2010 Conference Proceedings IPEC, Singapore, 27-29 October 2010, 842-847. https://doi.org/10.1109/IPECON.2010.5697083

[43] Boussaada, Z., Curea, O., Camblong, H., Bellaaj Mrabet, N. and Hacala, A. (2014) Multi-Agent Systems for the Dependability and Safety of Microgrids. Springer-Verlag, Berlin.

[44] Bui, V.H., Hussain, A. and Kim, H.M. (2016) A Multiagent-Based Hierarchical Energy Management Strategy for Multi-Microgrids Considering Adjustable Power and Demand Response. IEEE Transactions on Smart Grid, 9, 1323-1333.

[45] Musleh, A.S., Yao, G. and Muyeen, S.M. (2019) Blockchain Applications in Smart Grid-Review and Frameworks. IEEE Access, 7, 86746-86757.

https://doi.org/10.1109/ACCESS.2019.2920682

[46] Marzband, M., Yousefnejad, E., Sumper, A. and Dominguez-García, J.L. (2016) Real Time Experimental Implementation of Optimum Energy Management System in Standalone Microgrid by Using Multi-Layer Ant Colony Optimization. International Journal of Electrical Power and Energy Systems, 75, 265-274. https://doi.org/10.1016/j.ijepes.2015.09.010 
[47] Talari, S., Yazdaninejad, M. and Haghifam, M.R. (2015) Stochastic-Based Scheduling of the Microgrid Operation Including Wind Turbines, Photovoltaic Cells, Energy Storages and Responsive Loads. IET Generation, Transmission and Distribution, 9, 1498-1509. https://doi.org/10.1049/iet-gtd.2014.0040

[48] Farzan, F., Jafari, M.A., Masiello, R. and Lu, Y. (2015) Toward Optimal Day-Ahead Scheduling and Operation Control of Microgrids under Uncertainty. IEEE Transactions on Smart Grid, 6, 499-507. https://doi.org/10.1109/TSG.2014.2368077

[49] Surender Reddy, S. and Momoh, J.A. (2015) Realistic and Transparent Optimum Scheduling Strategy for Hybrid Power System. IEEE Transactions on Smart Grid, 6, 3114-3125. https://doi.org/10.1109/TSG.2015.2406879

[50] Lan, Y., Guan, X.H. and Wu, J. (2016) Rollout Strategies for Real-Time MultiEnergy Scheduling in Microgrid with Storage System. IET Generation, Transmission \& Distribution, 10, 688. https://doi.org/10.1049/iet-gtd.2015.0426 